\title{
On the Wind and Turbulence in the Lower Atmosphere above Complex Terrain
}

\author{
George Jandieri ${ }^{1}$, Alexander Surmava ${ }^{2}$, Anzor Gvelesiani ${ }^{2}$ \\ ${ }^{1}$ Georgian Technical University, Institute of Cybernetics, Tbilisi, Georgia \\ ${ }^{2}$ M. Nodia Institute of Geophysics, Tbilisi, Georgia \\ E-mail: jandieri@access.sanet.ge, aasurmava@yahoo.com \\ Received November 18, 2010; revised December 20, 2010; accepted December 23, 2010
}

\begin{abstract}
Numerical modeling and studies of the wind fields at the junction of three continents: over the complex terrains of the South-east Europe, Asia Minor, Middle East, Caucasus and over the Black, Caspian and Mediterranean seas have been carried out for the first time. Traveling synoptic scale vortex wave generation and subsequent evolution of orographic vortices are discovered. Wind fields, spatial distribution of the coefficients of subgrid scale horizontal and vertical turbulence and the Richardson number are calculated. It is shown that the local relief, atmospheric hydrothermodynamics and air-proof tropopause facilitate the generation of $\beta$-mesoscale vortex and turbulence amplification in the vicinity of the atmospheric boundary layer and tropopause. Also turbulence parameters distribution in the troposphere has the same nature as in the stratosphere and mesosphere: turbulence coefficients, stratification of the vertical profiles of the Richardson number, thickness of the turbulent and laminar layers.
\end{abstract}

Keywords: Numerical Modeling, Complex Terrain, Characteristics of Atmospheric Turbulence, Wind Field, Mesoscale Vortex, Bulk Richardson Number

\section{Introduction}

At present many hydrometeorologists are actively studying mesoscale atmospheric processes above the complex terrains. The problem is complex and its solution is closely related to other important problems of dynamical meteorology, including a problem of atmospheric turbulence of different scales in the mountainous regions.

Boundary-layer studies of mountainous terrain have mainly focused on global characteristics, such as thermal stratification, boundary-layer growth rates, circulation patterns and valley winds. The relatively few studies devoted to the turbulence structure over non-flat terrain have mainly been restricted to comparatively gentle hills. In [1], the nature of turbulent kinetic energy in a steep and narrow Alpine valley in Switzerland under fairweather summertime conditions was investigated for a detailed case study, in which the evaluation of aircraft data was combined with the application of high-resolution large-eddy simulations using the numerical model ARPS. Excellent correlation was established between surface heat flux and the up-valley wind speed. The measure- ments and simulations show that despite the complexity of the terrain and the apparent differences from a classical convective boundary layer, the turbulence structure reveals reproducible patterns and scaling characteristics. Generalization of obtained results by [1] requires an investigation of other valley geometries and different topographic orientations. In particular, further investigation of the functional dependence between up-valley wind speed and local surface fluxes. The authors of paper [2] studied vertical winds and turbulence in the troposphere in mountain-wave conditions near Kiruna in Arctic Sweden. They show that the horizontal and vertical wavelengths of the dominating mountain-waves were $\sim 10$ - 20 $\mathrm{km}$, the amplitudes in vertical wind $1-2 \mathrm{~m} \cdot \mathrm{s}^{-1}$, and turbulence velocities below $5500 \mathrm{~m}$ height were about $40 \%$ of the time during winter months. This is a much higher rate than the Advanced Research and Weather Forecasting model (WRF) predictions for conditions of Richardson number $R i<1$ but similar to WRF predictions of $R i<2$. The cause of low $R i$ is a combination of wind-shear at synoptic upper-level fronts and perturbations in static stability due to the mountain-waves. 
Comparison with radiosondes suggests that WRF underestimates wind-shear and the occurrence of thin layers with very low static stability, so that vertical mixing by turbulence associated with mountain waves may be significantly more than suggested by the model. A statistical correlation between enhanced turbulence and gravity waves was noted in [3].

The authors [4] investigating turbulence of a neutral atmosphere at different heights obtained the following results: 1) Radar observations of the mesosphere, aircrafts smoke trails into the stratosphere, radar observations of thin layers in the troposphere and lower stratosphere show that layered and stratified phenomena are common in the atmosphere. It was shown that "cat's-eye" structures are not uncommon in the atmosphere as a precursor to turbulence breakdown and such structures are often associated with Kelvin-Helmholtz (K-H) billows. Authors of [5] have indicated that other mechanism, such as the Holmboe instability, can also generate such structures. K-H instabilities are only dominant in weakly stratified flows, whereas the Holmboe instability is more likely in strongly stratified flows. There can be occasions when other mechanisms of breakdown can be responsible for the turbulence. The formation of cat's-eye structures is only one possible mechanism. Cat's-eye structures are visually impressive, so they tend to dominate the literature, but it is not clear whether they are in fact the main mode of turbulence breakdown at all. 2) By using radars and various specialized techniques they have also obtained information at smaller scales ( 1 m $100 \mathrm{~m}$ ).Turbulence is considered here to be generally the result of a non-linear breakdown of larger, more organized structures which have become unstable, with scales comparable to or larger than the buoyancy scale.

A number of remote-sensing wind-measuring instruments such as LIDAR and radar wind profilers [6] have been used for monitoring of low-level wind shear and turbulence. Data collected in a field experiment of the radio metering conducted in Hong Kong in February 2006 were used to obtain the Richardson number profile up to $1.5 \mathrm{~km}$ above ground. The Richardson number profiles are found to capture the turbulence events reasonably well. The atmospheric condition was favorable for the generation of turbulence when $R i<R i_{c}=0.25$. It was also noted that, at the above four times, the $R i$ number could be less than 0.25 below $400 \mathrm{~m}$ or so, especially the occurrence of negative $R i$ value. This feature is mainly related to turbulent airflow associated with thermodynamic instability near the ground. Downstream of the airflow, there are a number of waves/vortices between 700 and $1600 \mathrm{~m}$ high with the dimensions of several hundred meters in height and a couple of kilometers horizontally. A possible reason of turbulence is the shear instability associated with quick veering of the wind with height.

Meteorological researchers have made efforts to improve the stability description of a moist atmosphere by using variations of the Richardson number [7]. The Richardson number is often used as an important index for judging shearing instability and symmetry instability: the conventional baroclinic instabilities dominate if $R i>0.95$, symmetry instabilities dominate if $0.25<$ $R i<0.95$, and Kelvin-Helmholtz instabilities dominate if $R i<0.5$. A new Richardson number is defined for describing the stability of a moist atmosphere $\left(R i^{*}\right)$. The results show that convective instability is concentrated in the lower troposphere while instability determined by $R i^{*}<1$ is mainly located in the middle and upper troposphere above the rainfall areas, may be used to indicate and estimate the rainfall occurrence and development. Richardson numbers in the three thermodynamic states of the atmosphere can be obtained when potential temperature, $\theta$ (of dry atmosphere), equivalent potential temperature, $\theta_{e}$ (saturated moist region), and generalized potential temperature, $\theta^{*}$, are applied to calculate Brunt-Väisälä frequency, respectively.

In [8] spectral and structure function analyses of horizontal velocity fields observed in the upper troposphere and lower stratosphere during the Severe Clear Air Turbulence Collides with Air Traffic (SCATCAT) field program, conducted over the Pacific, were carried out to identify the scale interactions of turbulence and small scale gravity waves. In the presence of turbulence, transitional power spectra from $k^{-2}$ to $k^{-5 / 3}$ were found to be associated with the gravity waves and turbulence, respectively. The second-order structure function analysis was able to translate these spectral slopes into $r$ and $r^{2 / 3}$, consistent with Monin-Yaglom conversion law, in physical space, which presented clearer pictures of scale interactions between turbulence and gravity waves. The third-order structure function analysis indicated the existence of a narrow region of inverse energy cascade from the scales of turbulence up to the gravity waves scales. This inverse energy cascade region was linked to the occurrence of Kelvin-Helmholtz instability and other wave-amplifying mechanisms, which were conjectured to lead to the breaking of small-scale gravity waves and the ensuing generation of turbulence. The multifractal analyses revealed further scale breaks between gravity waves and turbulence. In [9] it is shown with a numerical simulation that a sharp increase in the vertical temperature gradient and Brunt-Väisälä frequency near the tropopause may produce an increase in the amplitudes of internal gravity waves propagating upward from the troposphere, wave breaking and generation of stronger turbulence. Turbulent diffusion coefficient calculated nu- 
merically and measured with MU radar are of $1-10$ $\mathrm{m}^{2} \cdot \mathrm{s}^{-1}$ in different seasons in Shigaraki, Japan $\left(35^{\circ} \mathrm{N}\right.$, $136^{\circ} \mathrm{E}$ ). These values lead to the estimation of vertical ozone flux from the stratosphere to the troposphere of $(1-10) \cdot 10^{14} \mathrm{~m}^{-2} \cdot \mathrm{s}^{-1}$ which may substantially add to the usually supposed ozone downward transport with the general atmospheric circulation. Therefore, local enhancements of IGW intensity and turbulence at tropospheric heights over the mountains due to their orographic excitation may lead to the changes in tropospheric and total ozone over different regions. In [10] high resolution $(150 \mathrm{~m})$ wind measurements by Mesosphere-Stratosphere-Troposphere (MST) radar and by Lower Atmospheric Wind Profiler (LAWP) have been used to study variation of turbulence intensity. Layers of higher turbulence are observed in the lower stratosphere. The heights of the turbulent layers in the lower stratosphere do not correlate with the levels of minimum Richardson number. During the short-period gravity wave activity $(\sim 7 \mathrm{~h})$ the high frequency convectively generated gravity waves breakdown generates the observed turbulence layers. A non-linear interaction between the waves of different scales might be responsible for the breakdown and generation of turbulence layers. It should be noted that different mechanisms of wave breaking can coexist and complement each other. From the above it follows that there are many unresolved problems related to the hydrodynamic interaction of air with a complex terrain.

The objective of this paper is theoretical investigation of the features of hydrodynamic interaction of the atmosphere with complex terrain at the junction of three continents: South-eastern Europe, Asia Minor and Africa, where there are located several large seas-the eastern part of the Mediterranean sea, the Black, Azov and Caspian seas, etc, many high mountain ranges, forests, steppes and deserts stretched a hundred kilometers. The evolution of the wave of cyclonic and anticyclonic vortices and the obtained mesoscale air flow for the considered complex terrain is modeled for the first time. We use a regional model of the atmospheric processes elaborated at the M. Nodia Institute of Geophysics [11].

\section{Formulation of the Problem}

\subsection{Basic Equations}

Basic equations of the model describing variations of the meteorological fields are:

1) for the troposphere $[12,13]$ :

$$
\begin{gathered}
\frac{\mathrm{d} u}{\mathrm{~d} t}=-\frac{\bar{P}}{\rho} \frac{\partial \phi}{\partial x}+l v+g(1+0.61 q) \vartheta \frac{\partial z}{\partial x}+\mu \Delta u+\frac{1}{\rho h^{2}} \frac{\partial}{\partial \zeta} \rho v \frac{\partial u}{\partial \zeta}, \\
\frac{\mathrm{d} v}{\mathrm{~d} t}=-\frac{\bar{P}}{\rho} \frac{\partial \phi}{\partial y}-l u+g(1+0.61 q) \vartheta \frac{\partial z}{\partial y}+\mu \Delta v+\frac{1}{\rho h^{2}} \frac{\partial}{\partial \zeta} \rho v \frac{\partial v}{\partial \zeta}, \\
\frac{\partial \phi}{\partial \zeta}=\frac{g}{R \bar{T}}(1+0.61 q) \vartheta h, \frac{\partial h}{\partial t}+\frac{\partial u h}{\partial x}+\frac{\partial v h}{\partial y}+\frac{\partial \tilde{w} h}{\partial \zeta}+\frac{1}{\rho} \frac{\mathrm{d} \rho}{d z} w h=0, \\
\frac{\partial \vartheta^{\prime}}{\partial t}+u \frac{\partial \vartheta}{\partial x}+v \frac{\partial \vartheta}{\partial y}+\tilde{w} \frac{\partial \vartheta}{\partial \zeta}+S w=\mu \Delta \vartheta+\frac{1}{\rho h^{2}} \frac{\partial}{\partial \zeta} \rho v \frac{\partial \vartheta}{\partial \zeta}+\frac{L}{\rho C_{p}} \varphi_{c o n}-\frac{\partial \theta}{\partial t}, \\
\frac{\partial q^{\prime}}{\partial t}+u \frac{\partial q}{\partial x}+v \frac{\partial q}{\partial y}+\tilde{w} \frac{\partial q}{\partial \zeta}=\mu \Delta q+\frac{1}{\rho} \frac{\partial}{\partial \zeta} \rho v \frac{\partial q}{\partial \zeta}-\varphi_{\text {con }}-\frac{\partial Q}{\partial t}, \Delta=\frac{\partial}{\partial x^{2}}+\frac{\partial}{\partial y^{2}}, \\
\frac{\partial m^{\prime}}{\partial t}+u \frac{\partial m}{\partial x}+v \frac{\partial m}{\partial y}+\tilde{w} \frac{\partial m}{\partial \zeta}=\mu \Delta m+\frac{\partial}{\partial \zeta} v \frac{\partial m}{\partial \zeta}+\varphi_{c o n}-\frac{\partial N}{\partial t}, \\
w=\frac{\partial z}{\partial t}+u \frac{\partial z}{\partial x}+v \frac{\partial z}{\partial y}+\tilde{w} h, \quad z=\zeta h+\delta, \frac{d}{d t}=\frac{\partial}{\partial t}+u \frac{\partial}{\partial x}+v \frac{\partial}{\partial y}+\tilde{w} \frac{\partial}{\partial \zeta},
\end{gathered}
$$

2) for the active layer of soil $[14,15]$ :

$$
\frac{\partial C}{\partial t}=\frac{\partial}{\partial z} D(C) \frac{\partial C}{\partial z}-\frac{\partial E(C)}{\partial z}, \frac{\partial T_{\text {soil }}}{\partial t}=K_{\text {soil }} \frac{\partial^{2} T_{\text {soil }}}{\partial z^{2}} \text { at } \delta_{0}>z>Z_{\text {soil }},
$$

3) for the layer of sea water [14]:

$$
\frac{\partial T_{\text {sea }}}{\partial t}=K_{\text {sea }} \frac{\partial^{2} T_{\text {sea }}}{\partial z^{2}}+\frac{1}{C_{\text {sea }} \rho_{\text {sea }}} \frac{\partial I}{\partial z}, \text { at } \delta_{0}>z>Z_{\text {sea }},
$$


where $t$ is time; $x, y$, and $z$ are the axes of the Cartesian coordinate directed to the east, north and vertically upwards, respectively; $\zeta=(z-\delta) / h$ is the dimensionless vertical coordinate; $\delta=\delta_{0}(x, y)+50 \mathrm{~m}$ is the surface layer height; $\delta_{0}$ is the height of the relief; $H(t, x, y)$ is the height of the tropopause; $h=H-\delta ; u, v, w$, and $w$ are the wind velocity components along the axes $x, y, z$, and $\zeta$, respectively; $\vartheta=T^{\prime} / \bar{T}$, and $\phi=P^{\prime} / \bar{P}(z)$ are the analogues of temperature and pressure, respectively; $\bar{T}=300 K ; T^{\prime}$ and $P^{\prime}$ are the devotions of temperature and pressure from the standard vertical distributions $T^{\prime}(t, x, y, z)=T(t, x, y, z)-\bar{T}+\gamma z-\overline{\bar{T}}(t, x, y, z)$, $P^{\prime}(t, x, y, z)=P(t, x, y, z)-\bar{P}(z)-\overline{\bar{P}}(t, x, y, z) ; T$ and $P$ are the temperature and pressure of the atmosphere, respectively: $\bar{T}-\gamma z$ and $\bar{P}(z)$ are the standard vertical distributions of the temperature and pressure, respectively; $\gamma$ is the standard vertical temperature gradient; $\overline{\bar{T}}$ and $\overline{\bar{P}}$ are the background deviations of the temperature and pressure from standard vertical distributions; $\vartheta$ and $\theta$ are the mesoscale and background components of the analogue of temperature, respectively; $\vartheta^{\prime}=\vartheta-\theta ; q$ and $Q$ are the mass fraction of water vapour and the background mass fraction of water vapour, respectively; $q^{\prime}=q-Q ; m$ and $M$ are the mass fraction of cloud water and the background mass of cloud water, respectively; $m^{\prime}=m-M ; T_{\text {soil }}$ and $T_{\text {sea }}$ are the temperatures of soil and seawater, respectively; $C$ is the volume content of soil water; $\rho(z)$ and $\rho_{\text {sea }}$ are the standard vertical distributions of the densities of dry air and seawater, respectively; $\sigma=-\rho^{-1} d p / d z ; g$ is the gravitational acceleration; $R$ is the universal gas constant for dry air; $C_{p}$ and $C_{\text {sea }}$ are the specific heat capacities of dry air at constant pressure and seawater, respectively; $S$ is the thermal stability parameter; $L$ is the latent heat of condensation; $\phi_{\text {con }}$ is the condensation rate; $\partial N / \partial t$ is the intensity of prescription; $\partial N / \partial t=\left(m-m_{c r}\right) / \delta t$ when $m>m_{c r}$, and 0 when $m \leq m_{c r}$ [14]; $m_{c r}$ is the critical magnitude of the mass fraction of a cloud water; $\delta t$ is the time of setting out of a surplus cloud water; $D$ is the diffusion coefficient of water in soil; $E$ is the filtration coefficient of water in soil; $I_{\text {sea }}$ is the total solar radiation flux in sea water; $K_{\text {soil }}$ and $K_{\text {sea }}$ are the thermal diffusivity coefficients of soil and sea water, respectively; $\mu$ and $v$ are the horizontal and vertical turbulent diffusion coefficients.

The set of Equations (1) and (2), (3) are solved in the coordinate systems $(t, x, y, \zeta)$ and $(t, x, y, z)$, respectively.The initial and boundary conditions, the values of background fields and methods of parameterization of the separate meteorological processes are selected in accordance with specific objectives of modeling.

\subsection{Boundary and Initial Conditions, the Main Parameters of the Regional Problem}

The boundary and initial conditions for the set of Equations (1-3) according to $[13,16,17]$ are:

\subsubsection{Vertical Boundary Conditions}

1) for the system (1):

$$
\begin{aligned}
& \frac{\partial \psi}{\partial \zeta}=0, \tilde{w}=0, \phi=\phi_{1}(t, x, y)+(g / R \bar{T}) \Delta \theta(h(t, x, y)-h(0, x, y)) \text {, at } \zeta=1 \text {, } \\
& v \frac{\partial u}{\partial \zeta}=A|V| u h / \Delta \zeta_{0}, \quad v \frac{\partial v}{\partial \zeta}=A|V| v h / \Delta \zeta_{0}, \\
& \left.v \frac{\partial \vartheta^{\prime}}{\partial \zeta}=A|V|\left(\vartheta^{\prime}-\vartheta_{0}^{\prime}\right) h / \Delta \zeta_{0}, \quad v \frac{\partial m^{\prime}}{\partial \zeta}=A|V| m^{\prime} h / \Delta \zeta_{0},\right\} \text { at } \zeta=0 \text {, } \\
& v \frac{\partial q^{\prime}}{\partial \zeta}=A|V|\left(q^{\prime}-q_{0}^{\prime}\right) h / \Delta \zeta_{0}, \quad \tilde{w}=0,
\end{aligned}
$$

2) for systems (2) and (3):

$$
\begin{gathered}
\rho_{e} C_{e} K_{e} \frac{\partial T_{e}}{\partial z}-\rho C_{p} A|V|\left(T-T_{e}\right) / \Delta \zeta_{0}-\rho L_{q} A|V|\left(q-q_{e}\right) / \Delta \zeta_{0}=I_{e} \\
C=C_{p o r}, \quad \text { at } \int_{0}^{1} \partial N / \partial t \mathrm{~d} \zeta>0 \\
D \frac{\partial C}{\partial z}=\frac{\rho_{w}}{\rho} A|V|\left(q-q_{e}\right), \quad \text { at } \int_{0}^{1} \partial N / \partial t \mathrm{~d} \zeta \leq 0 \\
\frac{\partial T_{e}}{\partial z}=\frac{\partial C}{\partial z}=0 \quad \text { at } \quad z=\delta_{0},
\end{gathered}
$$


where $|V|=\left(u^{2}+v^{2}\right)^{1 / 2} ; \phi_{1}$ is a given function of time and coordinate and shows the magnitude of the pressure in the tropopause; $\Delta \zeta_{0}$ is nondimensional thickness of the atmospheric surface layer; $\psi=\left(u, v, \vartheta^{\prime}, q^{\prime}, m^{\prime}\right)$; index " 0 " indicates the value of the function at the level $z=\delta_{0} ; \quad q_{0}^{\prime}=q_{0}-q_{\text {sat }}$ on the sea surface and $q_{0}^{\prime}=$ $\left(q_{0}-q_{s a t}\right) C / C_{\text {por }}$ on the soil surface; $C_{\text {por }}$ is the porosity of the soil, index " $e$ " indicates either "sea" or "soil" for the sea and soil surfaces; $C_{\text {soil }}$ and $\rho_{\text {soil }}$ are the specific heat capacity and soil density, respectively; $A$ and $\Delta \zeta_{0}$ are constant parameters.

\subsubsection{Lateral Boundary Conditions}

$u=\overline{\bar{u}}(t, x, y, \zeta), v=\overline{\bar{v}}(t, x, y, \zeta), h=\overline{\bar{h}}(t, x, y), \quad \vartheta^{\prime}=$ $q^{\prime}=m^{\prime}=0$ if vector $\mathbf{v}$ has inward direction inside a modeling area; in other cases the Von Neumann condition $\partial \psi / \partial n=\partial h / \partial n=0$ is used. Here $\overline{\bar{u}}, \overline{\bar{v}}$, and $\overline{\bar{h}}$ are the background values of the wind velocity components and atmosphere thickness, respectively; $n$ is a normal to the lateral boundary.

\subsubsection{Initial Conditions}

At $t=0$ we have:

3 ) for the system (1)

$$
\begin{gathered}
\vartheta^{\prime}=q^{\prime}=m^{\prime}=0, u=\overline{\bar{u}}(0, x, y, \zeta), v=\overline{\bar{v}}(0, x, y, \zeta) \\
\text { and } h=\overline{\bar{h}}(0, x, y)=9000-\delta(x, y) \mathrm{m},
\end{gathered}
$$

4) for Equations (2) and (3):

$$
C=C_{0}(x, y, z), T_{\text {sea }}=T_{0, \text { sea }}(x, y, z), T_{\text {soil }}=T_{0, \text { soil }}(x, y, z) \text {, }
$$

where $\overline{\bar{u}}$ and $\overline{\bar{v}}$ are determined by both geostrophic wind and quasi-static equations at the background com- ponents similar to the temperature and pressure at the tropopause level:

$$
\begin{gathered}
\overline{\bar{\theta}}(t, x, y, \zeta)=(6-3 \sin (\pi(x-10 t) /(568000) \sin (\pi y / 568000 Y)) /(288.15-0.065 z) \\
\phi_{1}=-0.012+0.0066 \sin (\pi(x-10 t) / 568000) \sin (\pi y / 568000)
\end{gathered}
$$

$C_{0}, T_{0, \text { sea }}$ and $T_{0, \text { sea }}$ are average monthly values for the June.

Coefficient of vertical turbulence decreased in the vertical direction from the value at the surface layer from 5 $\mathrm{m}^{2} \cdot \mathrm{s}^{-1}$ up to $0.001 \mathrm{~m}^{2} \cdot \mathrm{s}^{-1}$ at a height of $3-4 \mathrm{~km}$ above the Earth's surface. At more high altitudes equals to $0.001 \mathrm{~m}^{2} \cdot \mathrm{s}^{-1}$. Coefficient of the horizontal turbulence is equal to $5000 \mathrm{~m}^{2} \cdot \mathrm{s}^{-1}$. Background value of the relative humidity is equal to $40 \%$, the background value of the water content mass concentration zero. Other meteorological parameters are the well known values characterizing middle latitudes.

Numerical integration of Equations (1) and (2), (3) are carried out using [18] and the Crank-Nicolson schemes, respectively. Rectangular finite-difference grid with $96 \times$ $68 \times 17$ grid points having $40 \mathrm{~km}$ horizontal steps and the non-dimensional vertical step equals to $1 / 17$ is used. In soil and sea-water number of levels is equal to 20 , vertical step is $10 \mathrm{~cm}$, and temporal step equals to $4 \mathrm{~min}$. From Equation (7) follows that the background fields of temperature, pressure and wind are selected modeling horizontal displacement of the cyclonic and anticyclonic vortices having synoptic linear scale $5680 \mathrm{~km}$ moving from the west-east direction with the phase velocity 10 $\mathrm{m} \cdot \mathrm{s}^{-1}$. Figure 1 shows the fields of the surface background temperature of the air $T(t, x, y, 0)$, corresponding fields of the velocity at $t=0,48$ hours and the surface pressure field at the level $z=0$ and $t=0$ calculating for a plane surface of the Earth $\left(\delta_{0}=0\right)$. It is the idealized picture of large-scale wave slowly moving to the west usually observing in the atmosphere [19]. Temperature differences between the centers of anticyclonic and cyclonic vortices equal to $6^{\circ} \mathrm{C}$. Maximum surface wind speed amounts to $18 \mathrm{~m} \cdot \mathrm{s}^{-1}$. In 48 hours the vortex wave travels eastward at a distance equalling to $1400 \mathrm{~km}$, and anticyclonic vortex generates in place of cyclonic vortex and vice-versa.

\subsection{Boundary and Initial Conditions, the Main Parameters of the Mesoscale Problem}

$115 \mathrm{~km} \times 105 \mathrm{~km}$ territory is selected for investigation of the wind field spatial distribution and atmospheric turbulence, located on the east coast of the Black Sea in the vicinity of Batumi. It covers part of the Black Sea, Colchis plain, Guria and Pontic ranges. The height of the ranges attain to 2 - $3 \mathrm{~km}$ (Figure 2). Mesoscale task is solved by the set of Equations (1-3) and conditions (4-7) in which the solutions of the regional task are obtained for the mesoscale domain as background meteorological fields. Vertical turbulent flows of momentum, heat and humidity at $\zeta=0$ and the meteorological parameters are defined in a surface layer $\delta+h \geq z>\delta$ using the well-known formulae [20,21]: 


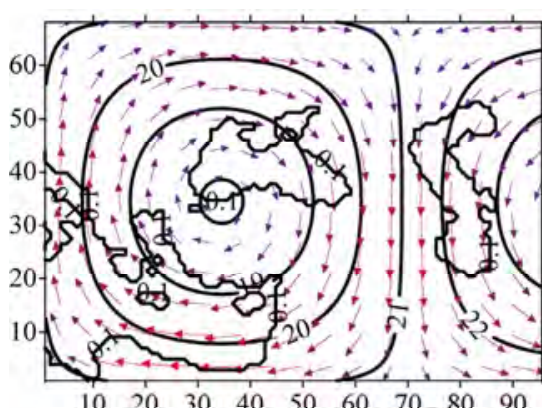

$\begin{array}{lllllllll}10 & 20 & 30 & 40 & 50 & 60 & 70 & 80 & 90\end{array}$

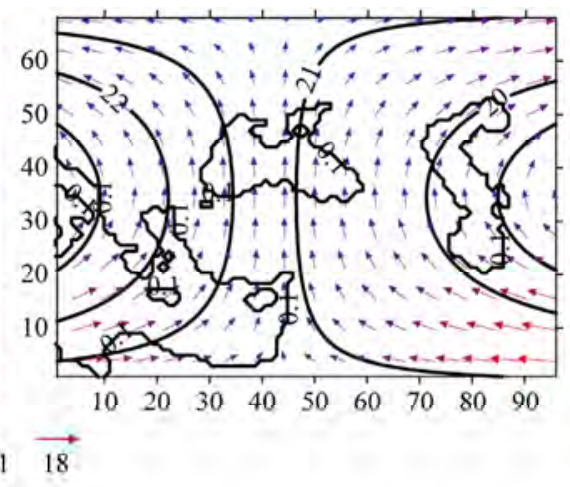

$0.1 \quad 18$

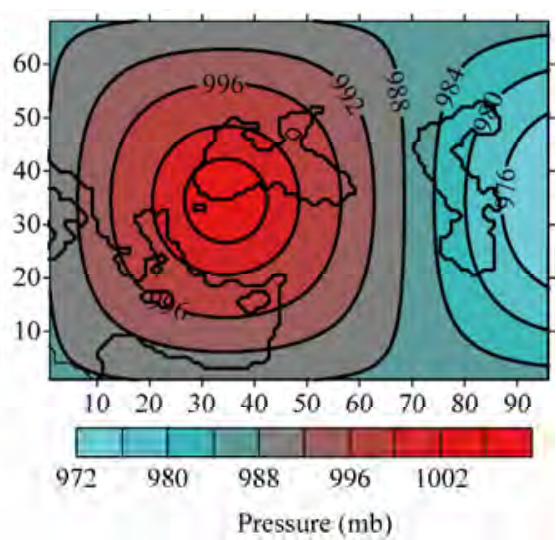

(c)

(a)

(b)

Figure 1. The background distributions of the wind velocity $\left(\mathrm{m} \cdot \mathrm{s}^{-1}\right)$, temperature (solid lines) $\left({ }^{\circ} \mathrm{C}\right)$ at $t=0 \mathrm{~h}$ (a) and $t=48 \mathrm{~h}$ (b), pressure (solid lines) fields (mb) at $t=0 \mathrm{~h}$ (c). Boundaries of the seas are shown by bold lines; horizontal and vertical grids steps are equal $40 \mathrm{~km}$.

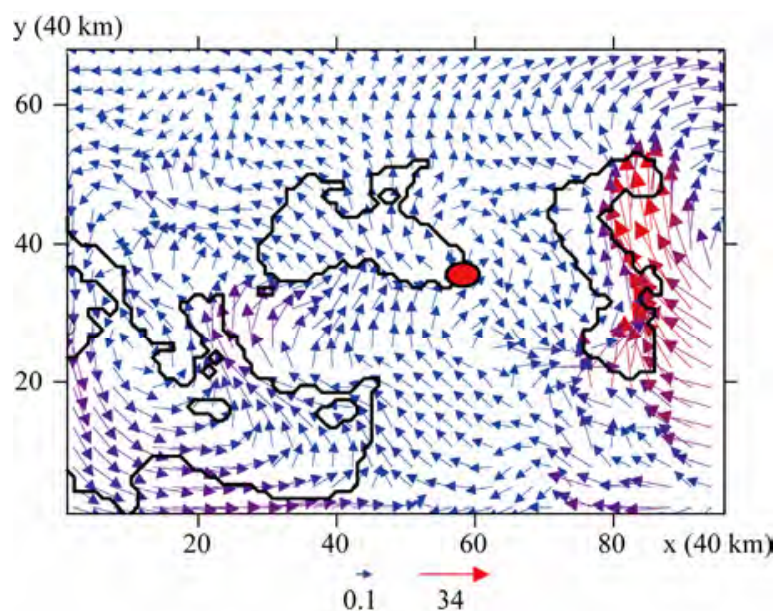

Wind velocity $(\mathrm{m} / \mathrm{s})$

(a)
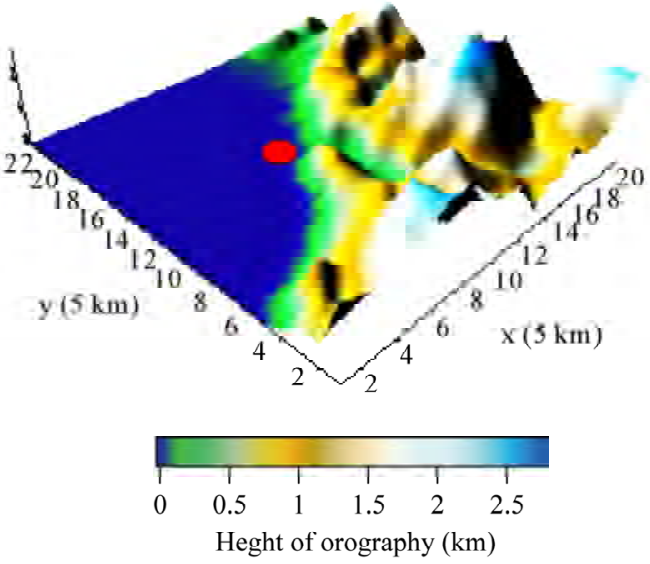

(b)

Figure 2. The background wind field distribution of the atmosphere surface layer for $t=72 h$ (a), and the topography of the mesoscale area around the Batumi port (b). The red circle shows the location of the Batumi port. Thick lines show the coastal lines of the seas; horizontal and vertical grids steps are equal $40 \mathrm{~km}$ (a) and $5 \mathrm{~km}$ (b), respectively.

$$
\mu=\Delta x \Delta y \sqrt{2\left(\frac{\partial u}{\partial x}\right)^{2}+\left(\frac{\partial u}{\partial y}+\frac{\partial v}{\partial x}\right)^{2}+2\left(\frac{\partial v}{\partial y}\right)^{2}} ; v=(0.05 \Delta z)^{2} \sqrt{\left(\frac{\partial u}{\partial z}\right)^{2}+2\left(\frac{\partial v}{\partial z}\right)^{2}-\frac{g}{\rho} \frac{\partial \rho}{\partial z}} \text {, when } z>h_{s}
$$

where $h_{s}$ is the height of the surface layer. If $z \leq h_{s}$, then the value of $v$ is determined by the parameteriza- tion method [22], based on the Monin-Obukhov similarity theory and solution of the following equations:

$$
\begin{gathered}
\frac{\partial|\mathbf{u}|}{\partial z}=\frac{u_{*}}{\chi z} \phi_{u}(\varsigma), \quad \frac{\partial p}{\partial z}=\frac{p_{*}}{\chi} \varphi_{\vartheta}(\varsigma), \quad(p=\vartheta, q), \quad \varsigma=\frac{z}{L}, \quad L=\frac{u_{*}^{2}}{\lambda \chi^{2} \vartheta_{*}^{2}} \\
|\mathbf{u}|=\frac{u_{*}}{\chi} f_{u}\left(\varsigma, \varsigma_{u}\right), \quad p-p_{0}=p_{*} f_{\vartheta}\left(\varsigma, \varsigma_{0}\right), \quad \varsigma_{u}=\frac{z_{u}}{L}, \quad \varsigma_{0}=\frac{z_{0}}{L}, \quad \text { if } \quad z \leq z_{s u r}, \\
v_{i}=\frac{u_{*} \chi z}{\phi_{i}(\varsigma)}, \quad\left(v_{i}\right)_{h}=\frac{u_{*} \chi h_{s}}{\phi_{i}\left(\varsigma_{h}\right)}(i=u, \vartheta), \quad \varsigma_{h}=\frac{h_{s}}{L},
\end{gathered}
$$


where $|\mathbf{u}|=\left(u^{2}+v^{2}\right)^{0.5}$ is the wind velocity; $u_{*}$ is the friction velocity; $\vartheta_{*}$ and $q_{*}$ are the scales of the potential temperature and mass fraction of water vapor, respectively; $\chi$ is the von Karman constant; $z_{0}$ and $z_{u}$ are the parameters of roughness for wind and temperature, respectively; $L$ is the length scale; $\lambda=g / \bar{T}$ is the parameter of convection; $\varphi_{u}(\varsigma), \varphi_{\vartheta}(\varsigma), f_{u}\left(\varsigma, \varsigma_{u}\right)$ and $f_{\vartheta}\left(\varsigma, \varsigma_{0}\right)$ are universal functions [22].

The background values of the meteorological fields for each time step were obtained in the process of modeling of the vortex wave propagation along the large-scale territory in the time interval of $96 \mathrm{~h} \leq t \leq 120 \mathrm{~h}$. South-east surface background wind is obtained at $t=96$ $\mathrm{h}$ in the vicinity of Batumi. The modeling is carried out by numerical integration of the set of Equations (1)-(5) and (9), (10) on the finite-difference grid $23 \times 21 \times 50$ with the grid points along the $x, y$ and $\zeta$ respectively. Horizontal steps were taken as equal to $5 \mathrm{~km}$, vertical non-dimensional step-equal to $1 / 50$ in the atmosphere, vertical step equal to $10 \mathrm{~cm}$ in the soil and sea water (with a number of levels equal to 20). Temporal step equaled to $1 \mathrm{~min}$.

\section{Simulation Results}

\subsection{The Results of Regional Model}

Calculations showed that in the barotropic approximation (in the absence of heat exchange between the underlying surface and atmosphere, $T^{\prime}=0$ and $S=0$ ), in case of the flat surface of the earth synoptic wave is stable and propagates eastward without perceptible variation with initial phase velocity of $10 \mathrm{~m} \cdot \mathrm{s}^{-1}$. In baroclinic approximation and at flat surface of the earth synoptic wave varies in shape and size in the process of traveling to the east, at the same time separate mesoscale (500$1000 \mathrm{~km}$ ) vortices of the wind velocity (Figure 3) generate and disappear. Life period of existing of these vortices is about 24-36 hours. Vortices are obtained not only at the surface of the Earth but also in the middle and upper troposphere. This indicates that there is an energy transfer from the large-scale vortex to the mesoscale vortices. The considered process takes place only in case of baroclinic atmosphere and is absent in barotropic case.

As follows from the Figure 4(a), complex relief significantly varies the general picture of large-scale movement of the air in the lower troposphere. Along with a dynamic baroclinic effect a kinematic effect of the relief lay on the motion of air. The mesoscale wave perturbation occurs at $t=0$ in the vicinity of the Carpathians as a result of the influence of orography. A zone of wind speed divergence is obtained in the Caucasus and northern Iran. The mesoscale cyclonic vortices generate in the vicinity of the eastern Black Sea and Mesopotamia. Strong north-west, north, northeast and east winds are obtained over the Caspian Sea, hilly areas of Iran and the Anatolian peninsula. A cyclonic circulation of wind was obtained over the relatively small territory to the east of the Caspian Sea. In general it is evident that the relief of the western and central parts of the region strengthens anticyclonic vorticity of the background movement of air. Figure 4(b) shows that as the background vortex propagates to the east, the surface flow varies significantly to the instant of time $t=24$ hours. Over the western region the anticyclonic vortex splits into medium-scale anticyclonic and cyclonic vortices with the centers in the vicinity of the Carpathians and the Crimea, respectively. Over the eastern part of the Mediterranean Sea wind was divided into two oppositely directed flows. Two countercurrent flow of air in the vicinity of Mesopotamia converge and form a strong southeast wind, which reaches the South Caucasus.

During the time interval of $\Delta t=48-72$ hours anticyclonic vortex in the vicinity of the Carpathians gradually defuses (Figures $\mathbf{4}(\mathbf{c}, \mathbf{d})$ ). In the vicinity of the Anatolian peninsula, the Caucasus and the Caspian Sea there are anticyclonic and cyclonic wind vortices. A cyclonic air movement over the Mediterranean Sea becomes stronger. After $t=96$ hours the process of propagation of large-scale wave vortex recurs with some quantitative differences of the meteorological fields. In general, the obtained wind fields at the level of the atmospheric surface layer qualitatively reproduce the fields that were constructed via the analysis of synoptic maps at the passage of large-scale pressure formations [23] over the Caucasus. The differences of the wind speeds and temperatures were calculated for the quantitative estimation of the relief influence effect, obtained taking into account the influence of orography irregularities and without taking it into account at the moment $t=24$ hours It was found that the influence of relief on the surface layer is manifested in reduction of large-scale cyclonic and anticyclonic vorticities. The wind speed in some parts of separate territory can vary by the value of the order of background wind speed, and the temperature-up to $10^{\circ} \mathrm{C}$. Thus, we can assume that the roughness of orography in the lower troposphere facilitates a development of mesoscale vortical turbulence followed by smoothing of the wind speed fields and temperature.

\subsection{Mesoscale Fields of the Wind Speed and Turbulence in the Troposphere}

Figure 5 shows spatial distributions of the wind field obtained at modeling of mesoscale circulation in the vicinity of city Batumi. From the figure it follows that the 


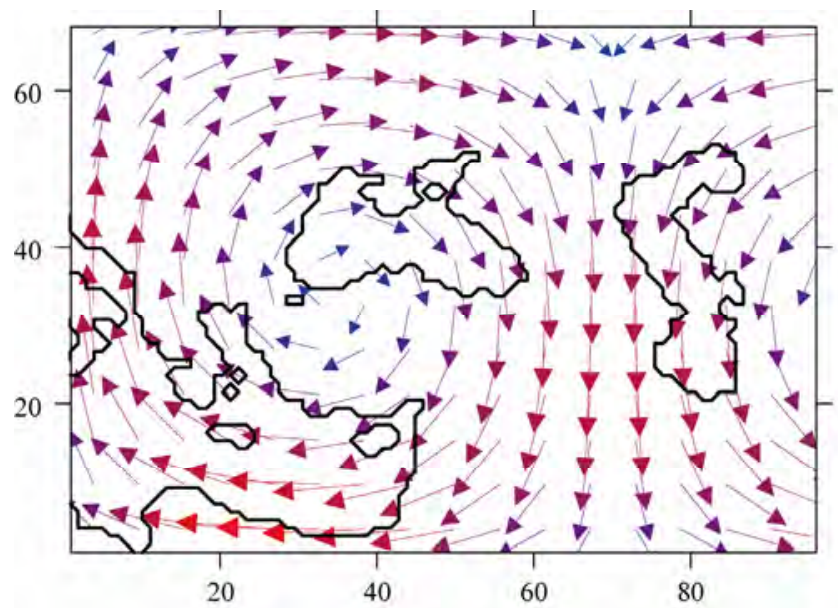

(a)

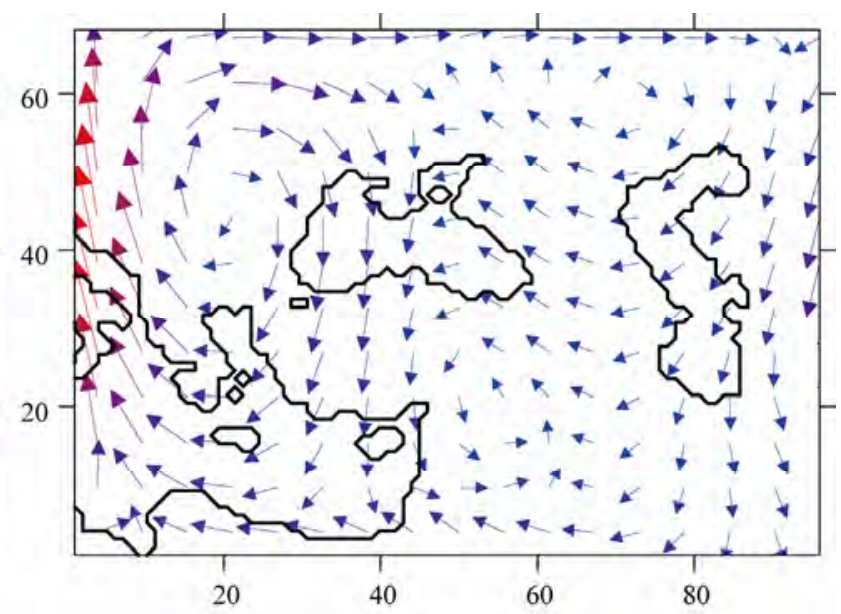

(b)

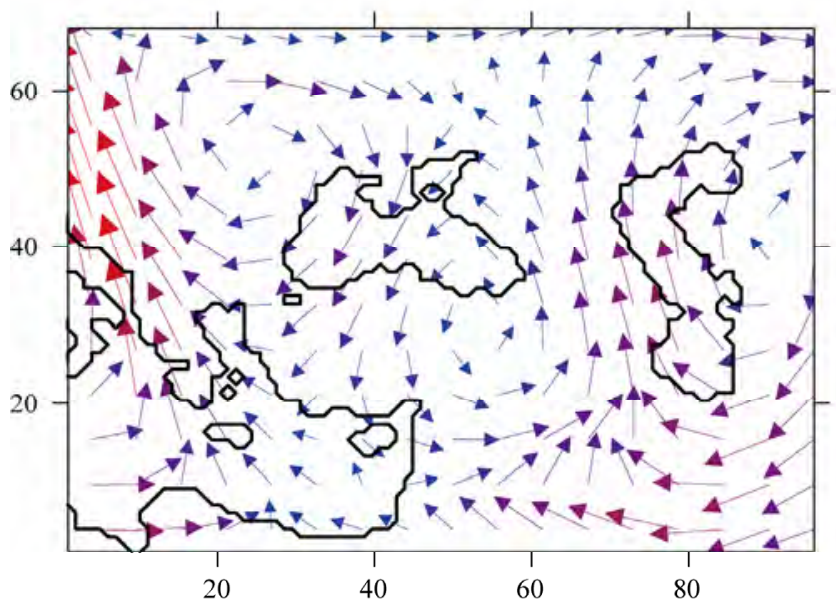

(c)

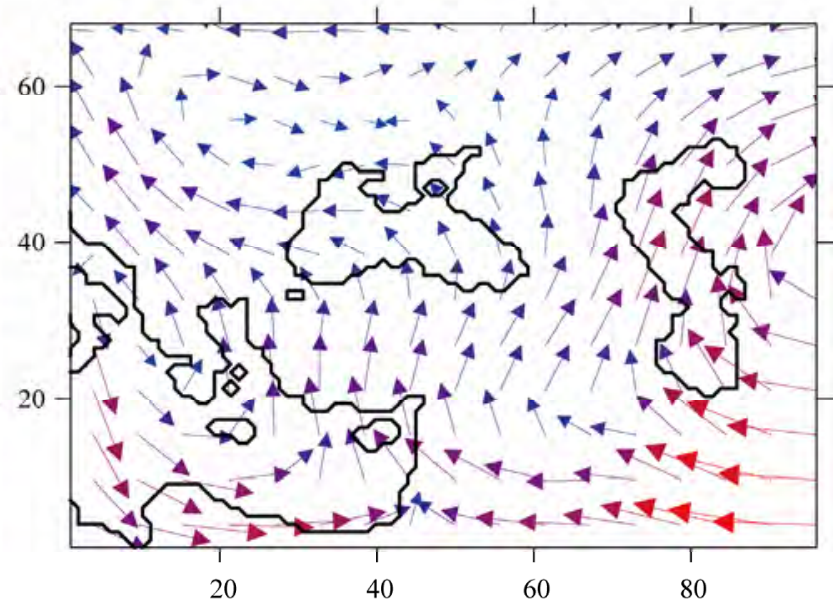

(d)

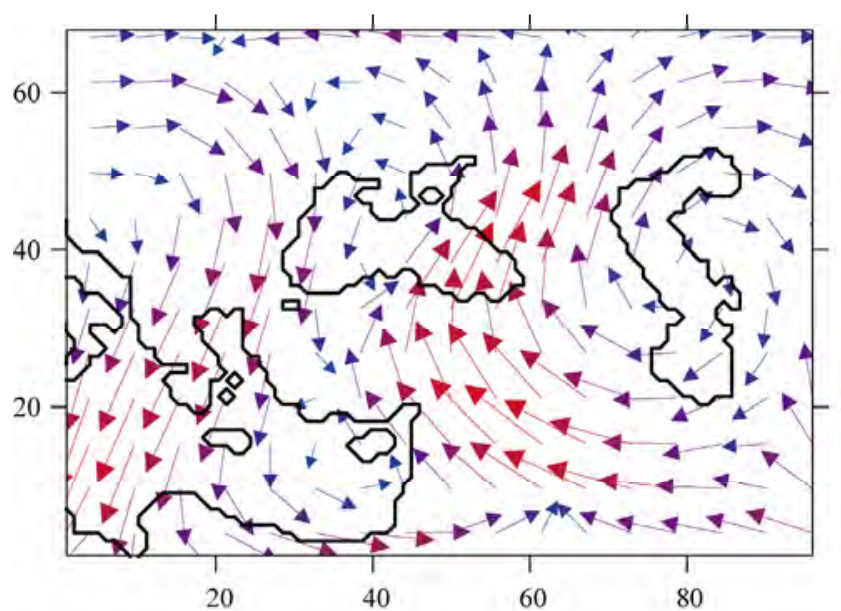

(e)

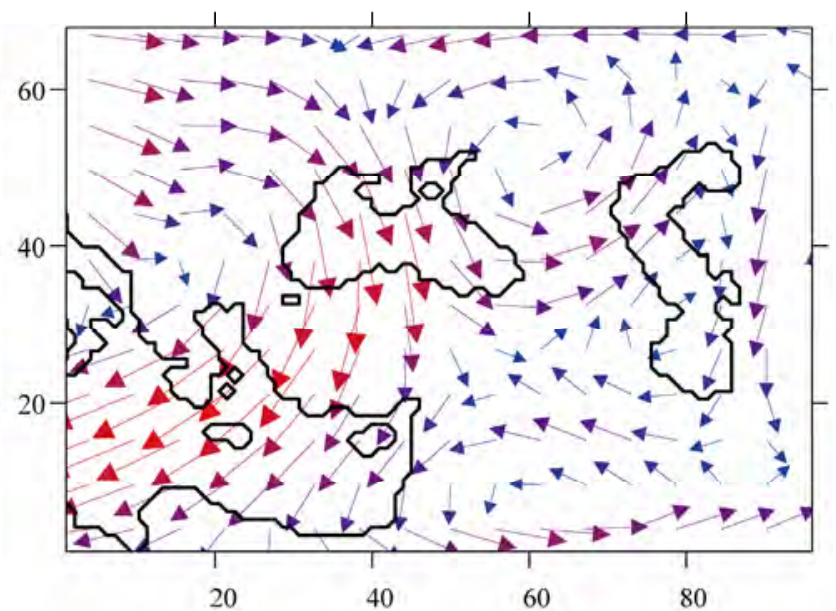

(I)

Wind velocity $(\mathrm{m} / \mathrm{s})$

Figure 3. The surface wind fields in the baroclyne approximation for a flat relief calculated at $t=0 h$ (a), $24 h$ (b), $48 h$ (c), 72 $h(d), 96 h$ (e), and 120 h (f), respectively. Horizontal and vertical grids steps are equal $40 \mathrm{~km}$. 


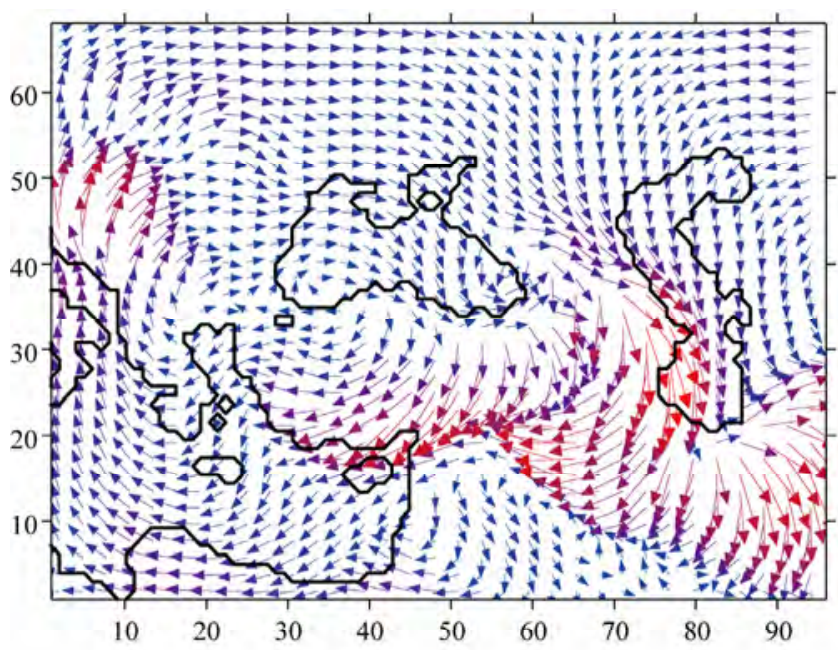

(a)

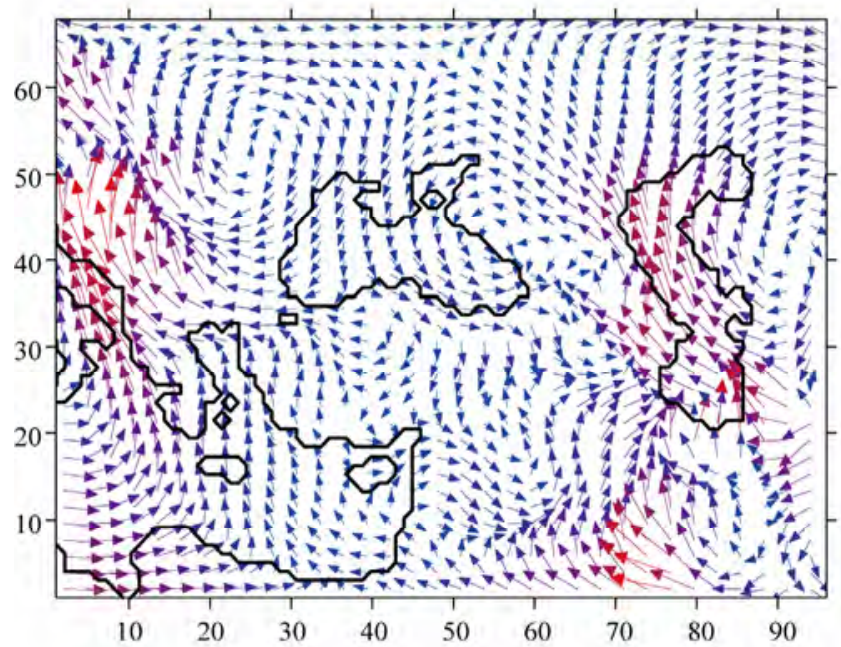

(c)

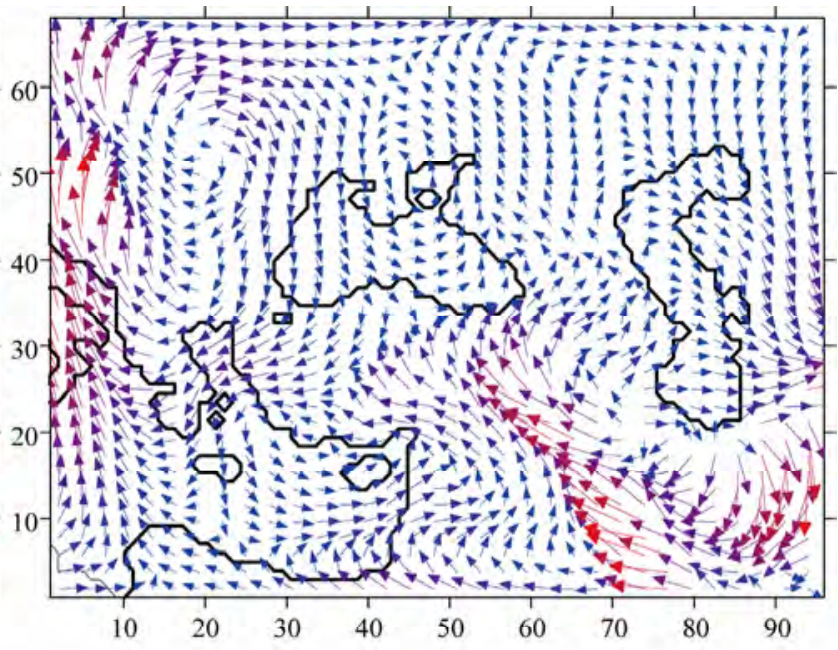

(b)

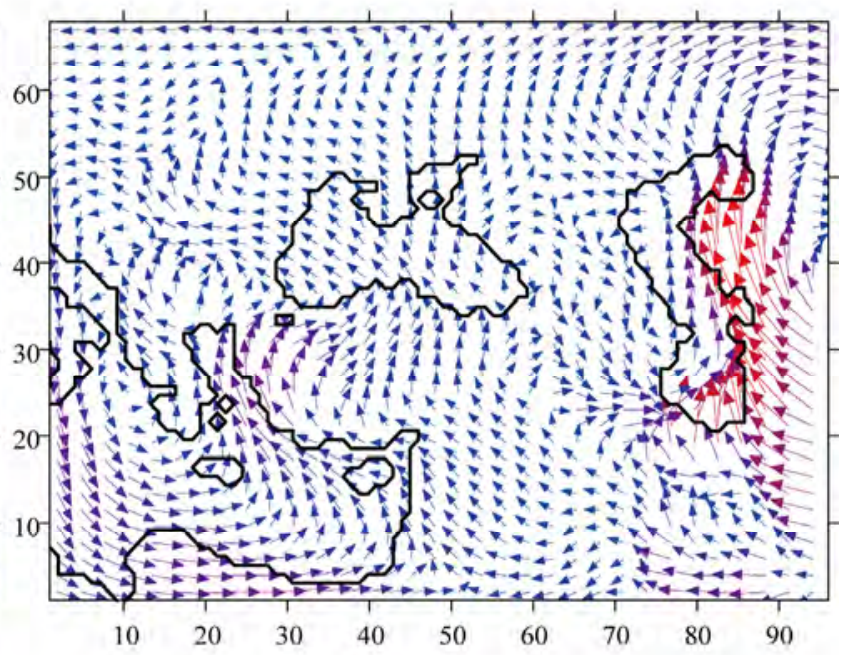

(d)

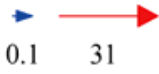

Wind velocity $(\mathrm{m} / \mathrm{s})$

Figure 4. The surface wind at $t=0 h$ (a), $24 h$ (b), $48 h$ (c), and 72 (d) $h$. Horizontal and vertical grids steps are equal $40 \mathrm{~km}$.

calculated field of wind speed in the lower and middle troposphere differs significantly from the background wind both by direction and magnitude. Northern, northwest and west winds were obtained at the surface layer $(z=50 \mathrm{~m})$. A closed mesoscale vortex is generated at the level $z=1000 \mathrm{~m}$ above the Black Sea. At the height of 2000 meters this vortex travels to the south-west. The wind direction varies gradually in the middle and upper troposphere. The wind direction approaches the background direction with removal from the Earth's surface. The analysis of the calculated wind field shows that the influence of relief is significant in the lower atmospheric layer having thickness 3-4 km.

Here the relief can substantially vary the direction and magnitude of the wind speed. The influence of tropopause dominates at the levels of the upper troposphere, where the wave disturbance lies on the background wind field. Figure 6(a) shows the diagrams of dependence of the vertical turbulence coefficient on the height above the earth's surface calculated at five nodal points of the modeling region. It is evident that the coefficients of the vertical turbulence are high in two regions: in the atmospheric boundary layer and in the upper troposphere (except for the item 5). Coefficients of the vertical turbulence are relatively small in troposphere at the heights 2.3 - $7 \mathrm{~km}$ from the earth surface.

The values of the horizontal turbulence coefficient are minimal at the heights of $500-1500 \mathrm{~m}$ above the earth 

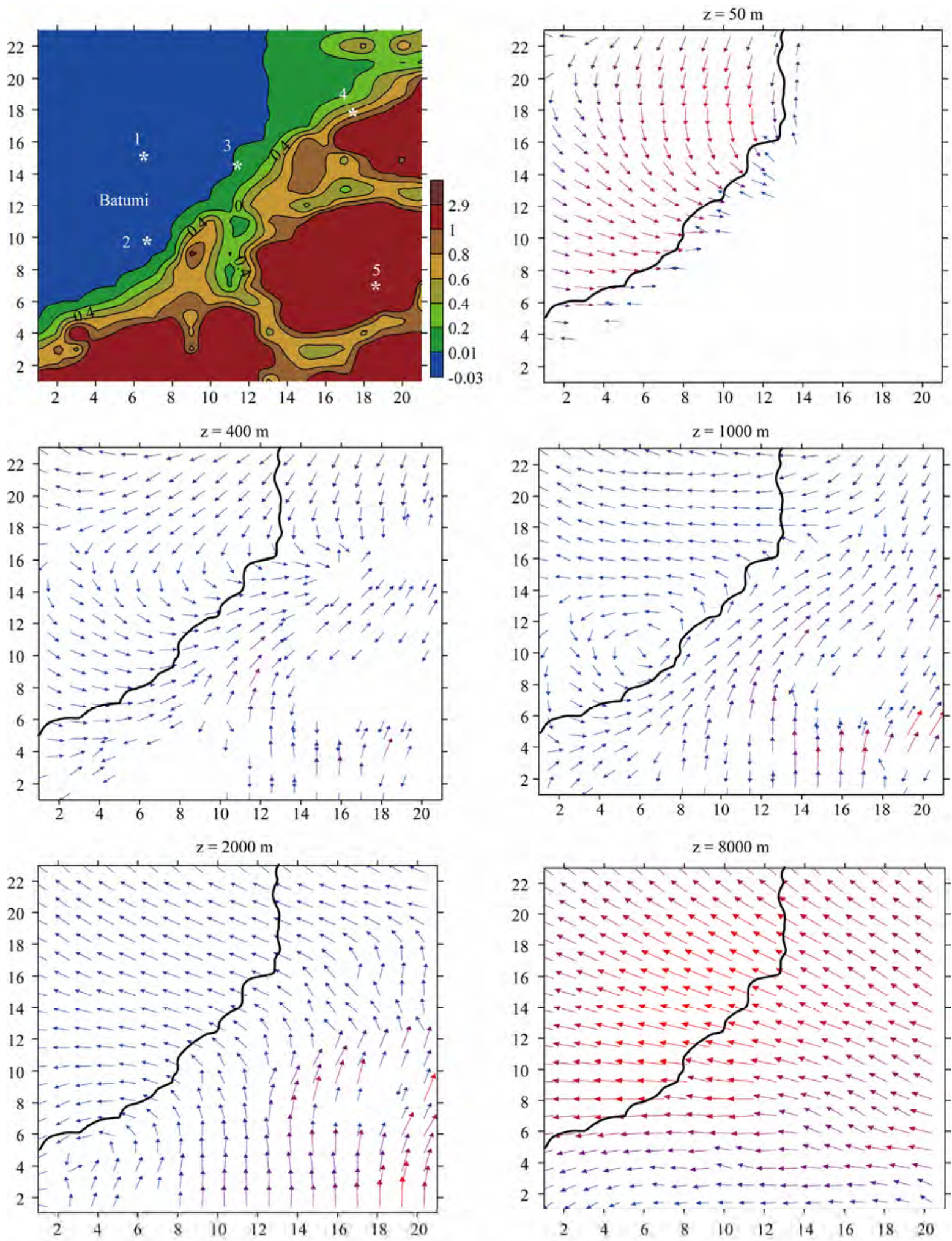

$\overrightarrow{0.1} \overrightarrow{12}$

Wind velocity $(\mathrm{m} / \mathrm{s})$

Figure 5. The topography and the wind fields at the altitudes $z=50 \mathrm{~m}, 400 \mathrm{~m}, 1000 \mathrm{~m}, 2000 \mathrm{~m}$, and $8000 \mathrm{~m}$, respectively. Horizontal and vertical grids steps are equal $5 \mathrm{~km}$. 


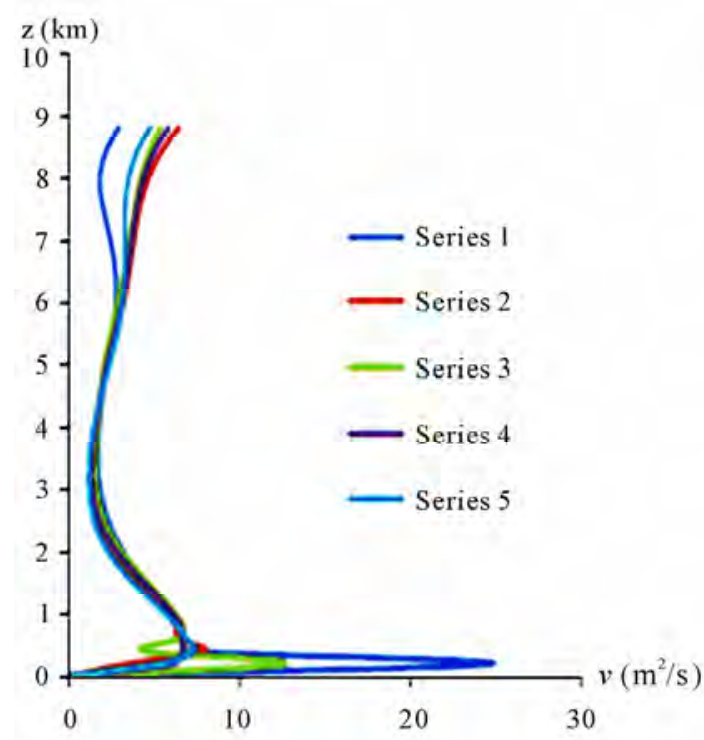

(a)

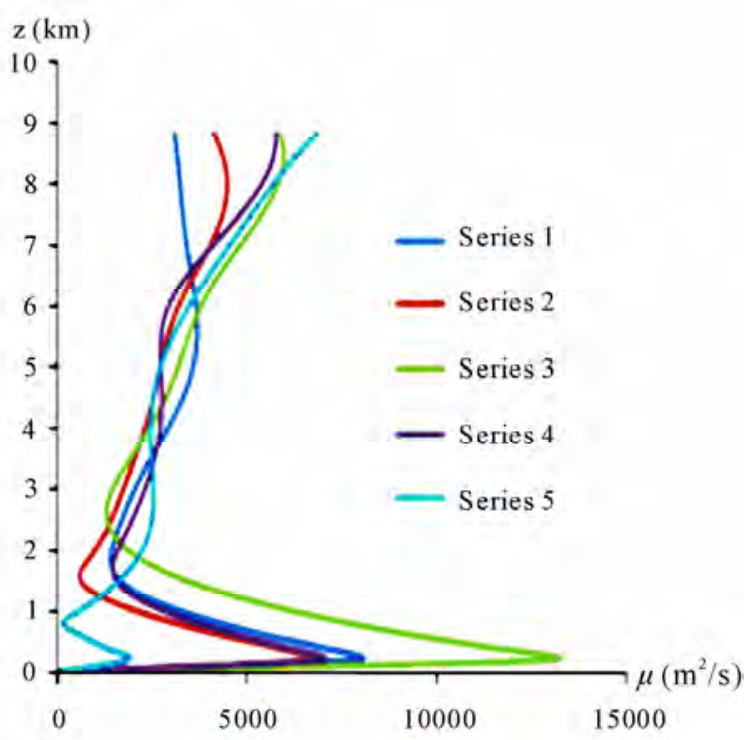

(b)

Figure 6. The profiles of the vertical $v\left(\mathrm{~m}^{2} \cdot \mathrm{s}^{-1}\right)$ (a) and horizontal $\mu\left(\mathrm{m}^{2} \cdot \mathrm{s}^{-1}\right)(\mathrm{b})$ turbulence coefficients for the points of the modeling area, respectively. Series $1,2,3,4$, and 5 correspond to the values of $v$ and $\mu$ over points 1, 2, 3, 4, and 5, respectively.

surface. The values of the horizontal turbulence gradually increase away from the middle troposphere, by 2.3 $7 \mathrm{~km}$ from the earth's surface. Qualitatively similar vertical distribution is obtained also for the coefficient of the horizontal turbulence (Figure 6(b)). The atmospheric boundary layer near the tropopause approaches to the values obtained in the surface layer. Thus impenetrable for air tropopause has almost the same influence on the horizontal turbulence as the Earth's surface. It should be noted that the calculated vertical turbulence coefficients are in agreement with the measurements data [9].

Figure 7 shows horizontal distribution of the vertical turbulence. Coefficient of the vertical turbulence is maximal in the surface layer in which they vary in the interval $0 \leq v \leq 48 \mathrm{~m}^{2} \cdot \mathrm{s}^{-1}$.

The values are minimal at the height of $z \sim 2-4 \mathrm{~km}$ from the Earth's surface. At this level their values do not exceed $2.5 \mathrm{~m}^{2} \cdot \mathrm{s}^{-1}$. At higher levels $v$ increases again and reaches peak values near the tropopause. Similar spatial distribution is obtained also for the horizontal turbulence (Figure 8). From these figures follow that atmospheric turbulence above the sea surface is less than above the earth surface. Figure 9 shows vertical profiles of the bulk-Richardson number $B R N=(g / \bar{T})$ $\left(\gamma_{a}-\gamma+\Delta\left(T^{\prime}+\overline{\bar{T}}\right) / \Delta z\right) /\left[(\Delta u / \Delta z)^{2}+(\Delta v / \Delta z)^{2}\right]$. The greatest values of this number are obtained in the atmospheric layer having thickness 3-6 km above the Earth's surface. In this layer vertical gradient of the wind speed is relatively small, the Richardson number is much greater than 45 and the atmosphere is stratified stably.
Air flow in the mesoscale region is a part of the $\alpha$ mesovortex (Figure 4(a)) generated due to the interaction of the Caucasus relief with the background wind. Vertical distribution of the meteorological parameters varies in the atmospheric boundary layer due to the thermal and dynamic influence of the Earth's surface. BRN numbers in the narrow layers between the heights of $500 \mathrm{~m}$ and $2500 \mathrm{~m}$ take on the values in the interval 10 - 45. These values of the BRN correspond to the vortex generation conditions $[24,25]$ and formation of the $\beta$ mesoscale vortex (Figure 5, $z=1000 \mathrm{~m}$ and $2000 \mathrm{~m}$ ). From the analysis of the Figure $\mathbf{1 0}$ follows that changing of the Richardson BRN number in time at different heights of the surface layer $(z \leq 50 \mathrm{~m})$ is periodical with a period of 12 - 26 hours. At certain hours (50-60 h) the BRN number substantially decreases and can become less than 0.25 at which small-scale turbulence should generate. The obtained picture of the temporal dependence of the BRN is qualitatively consistent with the results of [2] (see Figure 9).

\section{Discussion}

The performed calculations showed the peculiarities of the influence of large-scale relief on formation of the wind fields and vortex turbulence. In particular, it is shown that the influence of relief on the synoptic scale movement help generate of orographic vortices. The obtained orographic vortices are generated in the lower troposphere and their sizes can vary from a few hundred 

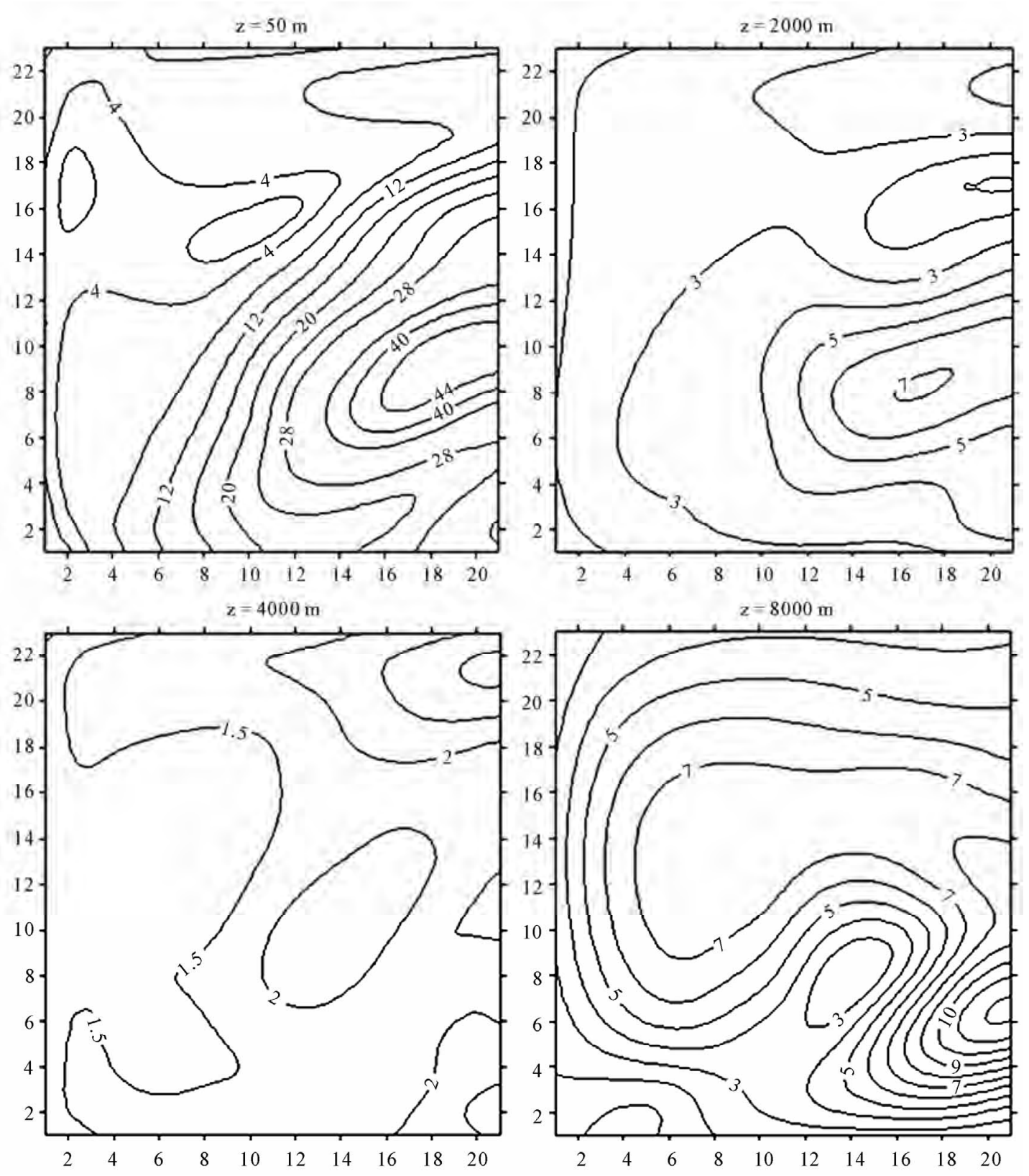

Figure 7. The horizontal distribution of the vertical turbulence coefficient $v\left(\mathrm{~m}^{2} \cdot \mathrm{s}^{-1}\right)$ at the altitudes $z=50 \mathrm{~m}, 2000 \mathrm{~m}, 4000 \mathrm{~m}$, and $8000 \mathrm{~m}$, respectively. Horizontal and vertical grids steps are equal $5 \mathbf{k m}$.

kilometers up to $1000 \mathrm{~km}$ and more. Analysis of the Figures $\mathbf{3}$ and $\mathbf{4}$ show that evidently the large-scale perturbations become smoother due to the energy transfer from the large-scale perturbations to the smaller-scale ones.

The main territories favorable for the generation of orographic vortices are mountainous areas adjacent to seas. High mountain ranges of the Caucasus, Anatolian peninsula and Asia Minor facilitate formation of medium-scale zones of the wind speed divergence.

The bands of relatively narrow and long zones in which the wind speed exceeds $20 \mathrm{~m} \cdot \mathrm{s}^{-1}$ are generated above the flat grounds between the mountain ranges. Due to these airflows the warm air of the Asia Minor can propagate far to the north up to the main Caucasian range (Figure 4(b)). The relief of the Caucasus is a natural barrier to the southern wind. However, the north wind may flow around the Caucasus from the Caspian Sea and propagate to the south, reaching the shores of the Mediterranean Sea (Figure 4(a)). Such picture is often observed in the Caucasus, above the Black and Caspian seas, especially in summer [26-28]. 

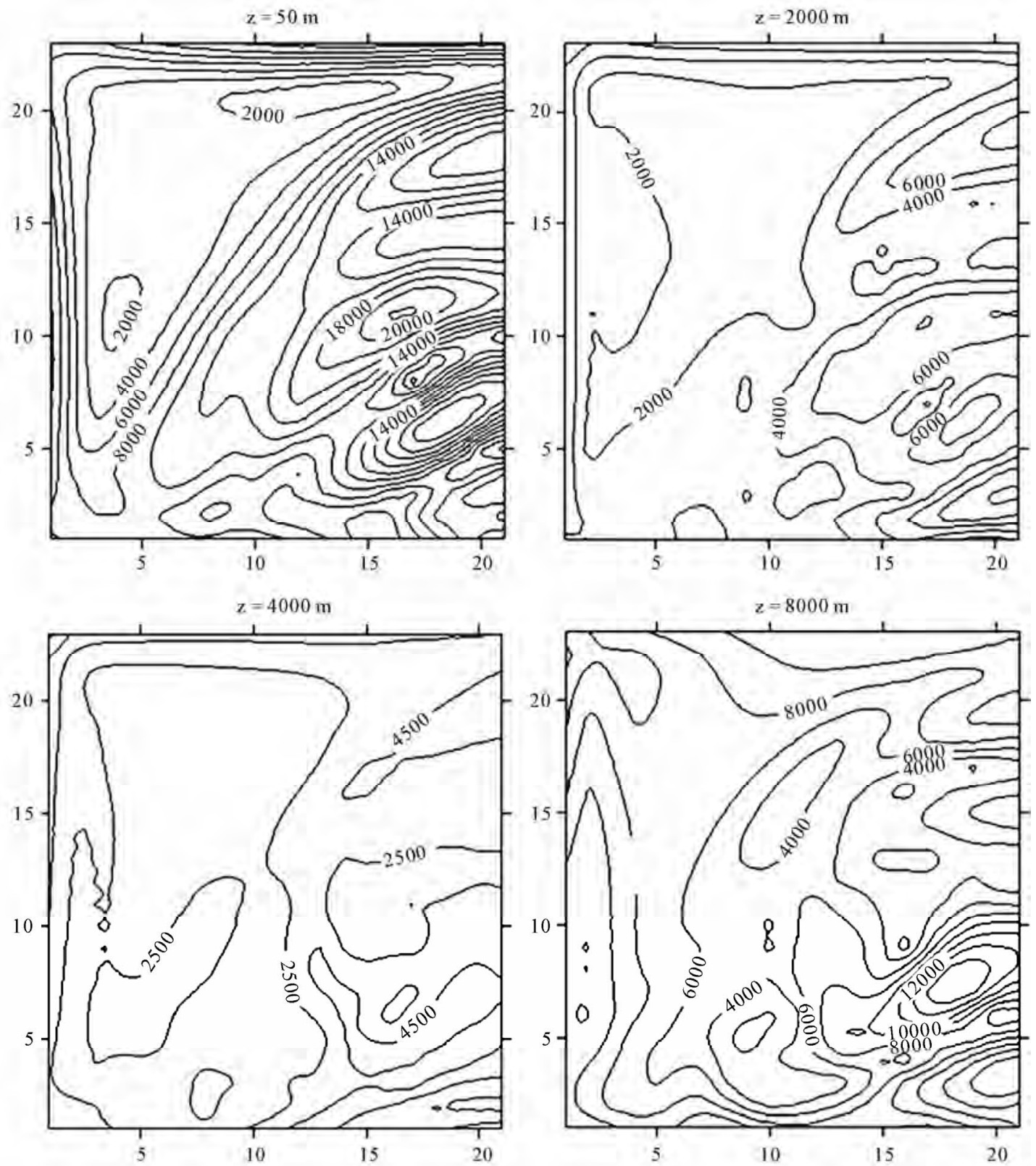

Figure 8. The horizontal distribution of the horizontal turbulence coefficient $\mu\left(\mathrm{m}^{2} \cdot \mathrm{s}^{-1}\right)$ at the altitudes $\mathrm{z}=50 \mathrm{~m}, 2000 \mathrm{~m}, 4000$ $\mathrm{m}$ and $8000 \mathrm{~m}$, respectively. Horizontal and vertical grids steps are equal $5 \mathrm{~km}$.

At investigation of mesoscale wind field structure with a horizontal 5-km step of grid it has been found that the wind field can substantially differ from the large-scale field if the background wind speed in the boundary layer does not exceed $5 \mathrm{~m} \cdot \mathrm{s}^{-1}$. In particular, a closed vortex with a diameter of 25 - $30 \mathrm{~km}$ (Figure 5) is generated at the height of about $1000 \mathrm{~m}$ near the southeastern coast of the Black Sea. Evidently such vortex can facilitate generation of a whirlwind, which is often observed on the Black Sea coast of Georgia [26] in summer.

Mesoscale roughness of relief exerts influence on the spatial distribution of the turbulence coefficients (Figures 7 and 8). It is obtained that in the atmospheric boundary layer over the sea surface the values of horizontal and vertical turbulence coefficients are several times smaller than the values obtained over rough Earth's surface. The more is the height of the area and slope of the earth surface the more is the difference. In the middle troposphere (2 - $6 \mathrm{~km}$ ) the difference between them (over the sea and the earth) practically disappears and reappears again near the tropopause $(8,9 \mathrm{~km})$ but to a lesser extent. Profiles of horizontal and vertical turbulence coefficients 


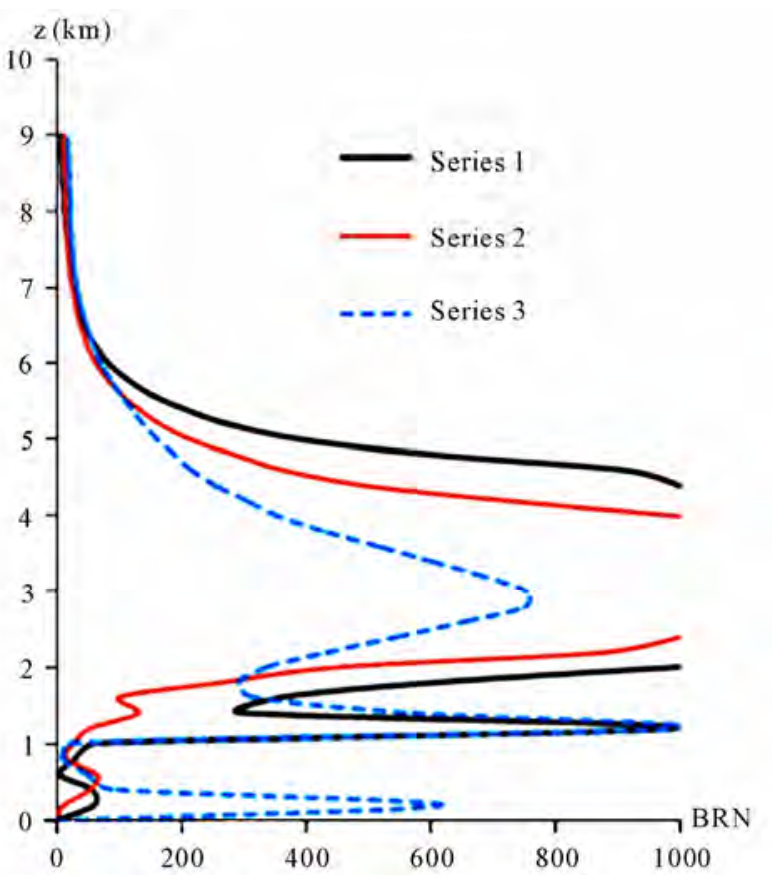

Figure 9. The profiles of Bulk-Richardson number (BRN) over 3 points of the area modeling. 1, 2, and 3 correspond to points 1, 2, and 3 (see Figure 5 (a)), respectively.

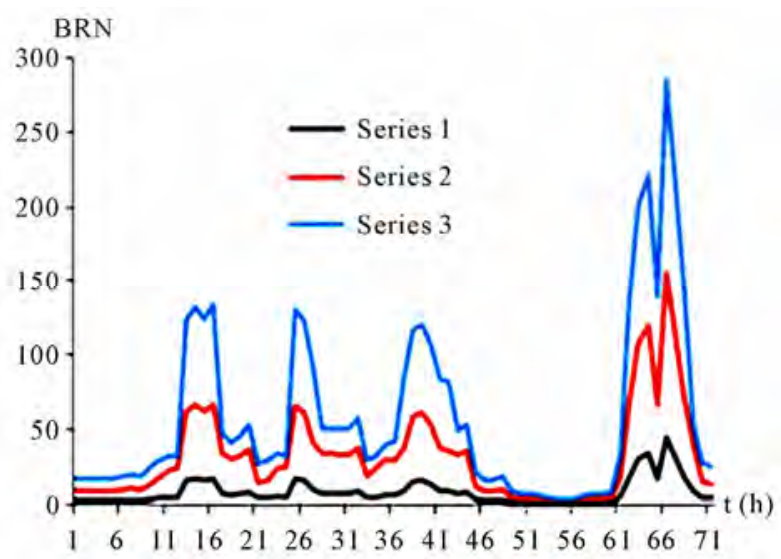

Figure 10. Time variability of BRN at the altitudes $z=2 \mathrm{~m}$ (series 1), $10 \mathrm{~m}$ (series 2) and $50 \mathrm{~m}$ (series 3) over point 2 of the Figure 5.

have the minimums in the atmospheric layer having thickness 2-6 km (Figure 6).

Qualitative similarity temporal dependence of the Richardson numbers (during three days) obtained above (Figure 10) and in [2] (Figure 9) is explained by the similarity of the relief and meteorological conditions (Figure 4) and (Figures 1 and 3 in [2]). This allows us to judge the validity of the obtained results. In the atmospheric boundary layer the dynamic state of the environment becomes favorable $(10 \leq B R N \leq 45)$ for the generation of a vortex cell (Figure 5) at certain moments.
The obtained vortex cell can not develop more and convert into a damp convection system due to the low background relative humidity (40\%).

Multistratification of the obtained vertical profiles of the turbulent parameters of the medium (with a layer thickness of $0.1-3 \mathrm{~km}$ ) is also observed in [1] for the complex relief, in the laboratory experiments modeling the process of convective instability in a stratified fluid [29] and at higher levels of the atmosphere (stratosphere and mesosphere) [30-32]. This means that there is much common in the turbulent features of the troposphere, stratosphere and mesosphere: the order of the turbulence coefficients, stratification of the vertical profiles of the Richardson numbers and alternation of the turbulent and laminar layers of the atmosphere.

\section{Conclusions}

Numerical investigation of the large-scale wind fields at the junction of three continents has been carried out for the first time: over the complex territories of the Southeast Europe, Asia Minor and Southwest Asia, Caucasus, Middle East and the water areas of the Black, Caspian and Mediterranean seas. By the example of a traveling synoptic-scale ( 6000 km) vortex wave generation and subsequent evolution of orographic vortices of the order of $1000 \mathrm{~km}$ are considered.

The mesoscale structures of hydrodynamic fields in the eastern coastal part of the Black Sea are modeled. On the mesoscale area $(\sim 100 \mathrm{~km})$ of the generated mediumscale vortex flow in the vicinity of the western Black Sea coast, hydrodynamic structure of the wind is studied by the nested grids method. Wind fields, spatial distribution of the coefficients of subgrid scale horizontal and vertical turbulence and the Richardson number (Bulk Richardson Number, BRN) are calculated. It is shown that the local relief, atmospheric hydrothermodynamics and air-proof tropopause facilitate the generation of $\beta$ - mesoscale vortex and turbulence amplification in the vicinity of the atmospheric boundary layer and tropopause. Minimum values of the turbulence coefficients were obtained at the heights between $2300 \mathrm{~m}$ and $4000 \mathrm{~m}$ above the earth's surface.

There is much in common in the nature of turbulence parameters distribution calculated for the troposphere and the well-known results of the observations in the stratosphere and mesosphere: the order of the turbulence coefficients, stratification of the vertical profiles of the Richardson number and alternation of about 0.1-3 km thickness turbulent and laminar layers.

Calculations showed the features of large- and mesoscale terrains influence on generation of the wind fields and vortex turbulence: 
- Large and high mountain ranges of the Caucasus, Minor and Southwest Asia facilitate formation of medium-scale zones of wind speed divergence and vortex structures;

- The main favorable territories for the generation of orographic vortices are the neighborhoods of the Carpathian Mountains and the Black, Caspian and Mediterranean seas;

- The orographic vortices are generated in the lower troposphere and their sizes can vary from a few hundred kilometers to 1 thousand kilometers and higher;

- Atmosphere tends to a smoother distribution of the large-scale meteorological fields due to the orographic vortices;

- Separate $\beta$-mesoscale vortices can generate against the background of the $\alpha$-mesoscale vortices in the atmospheric boundary layer in the vicinity of the sea-land at the low values of the vertical wind speed gradients;

- The underlying surface and impenetrability of tropopause equally facilitate turbulence amplification of the atmosphere;

- There are 0.1-3 km thickness turbulent stratifications in the troposphere similar as in $10 \mathrm{~km}$ layers of stratosphere and middle atmosphere;

- Vertical profiles of the Richardson numbers and the turbulence coefficients contain several (3-5) extremums.

The objective of the further investigation is specification of the atmosphere turbulization mechanisms, generation and evolution of the vortex structures over the considered complex terrain in the large and mesoscale hydrodynamic processes.

\section{References}

[1] A. P. Weigel, F. K. Chow and M. W. Rotach, "On the Nature of Turbulent Kinetic Energy in a Steep and Narrow Alpine Valley," Boundary-Layer Meteorology Springer Science-Business Media B.V., Vol. 123, 2006, pp. 177-199. doi:10.1007/s10546-006-9142-9

[2] S. Kirkwood, M. Mihalikova, T. N. Rao and K. Satheesan, "Turbulence Associated with Mountain Waves over Northern Scandinavia-a Case Study Using the ESRAD VHF Radar and the WRF Mesoscale Model," Atmospheric Chemistry and Physics Discussions, Vol. 9, No. 5, 2009, pp. 20775-20817. doi:10.5194/acpd-9-20775-2009

[3] G. D. Nastrom, K. S. Gage and W. L. Ecklund, "Variability of Turbulence, 4 - $20 \mathrm{~km}$, in Colorado and Alaska from MST Radar Observations,” Journal of Geophysical Research, Vol. 91, 1986, pp. 6722-6734. doi:10.1029/JD091iD06p06722

[4] W. K. Hocking and J. Röttger, "The Structure of Turbulence in the Middle and Lower Atmosphere Seen by and
Deduced from MF, HF and VHF Radar, with Special Emphasis on Small-Scale Features and Anisotropy," Annales Geophysicae, Vol. 19, 2001, pp. 933-944. doi:10.5194/angeo-19-933-2001

[5] W. D. Smyth and W. R. Peltier, "The Transition between Kelvin-Helmholtz and Holmboe Instability: An Investigation of the Over-Reflection Hypothesis," Journal of the Atmospheric Sciences, Vol. 46, No. 24, 1989, pp. 36983720 .

doi:10.1175/1520-0469(1989)046<3698:TTBKAH>2.0.C $\mathrm{O} ; 2$

[6] P. W. Chan, "Determination of Richardson number Profile from Remote Sensing Data and Its Aviation Application," Proceedings $14^{\text {th }}$ International Symposium for the Advancement of Boundary Layer Remote Sensing, IOP Conf. Series: Earth and Environmental Science 1, 012043, 2008. doi:10.1088/1755-1307/1/1/012043

[7] Yu. Zhao, X. P. Cui and S. Gao, "Richardson Number in a Moist Atrmosphere and Its Application in the Analysis of Heavy Rainfall Events," Acta Meteorologica Sinica, Vol. 24, 2010, No 1, pp. 95-103.

[8] C. G. Lu and S. E. Koch, "Interaction of Upper-Tropospheric Turbulence and Gravity Waves Obtaned from Spectral Structure Function Analyses," Journal of the Atmospheric Sciences, Vol. 65, No. 8, 2008, pp. 26762690. doi:10.1175/2007JAS2660.1

[9] N. M. Gavrilov and I. S. Fukao, "Numerical and the MU Radar Estimations of Gravity Wave Enhancement and Turbulent Ozone Fluxes Near the Tropopause,” Annales Geophysicae, Vol. 22, No. 11, 2004, pp. 3889-3898 and p. SRef-ID: 1432-0576/ag/2004-22-3889.

[10] G. Dutta, M. C. A. Kumar, P. V. Kumar, P. V. Rao, B. Bapiraju and H. A. Basha, "High Resolution Observations of Turbulence in the Troposphere and Lower Stratosphere over Gadanki,” Annales Geophysicae, Vol. 27, No. 6, 2009, pp. 2407-2415. doi:10.5194/angeo-27-2407-2009

[11] A. A. Kordzadze, A. A. Surmava, D. I. Demetrashvili, and V. G. Kukhalashvili, "Numerical Investigation of the Influence of the Caucasus Relief on the Distribution of Hydrometeorological Fields," Izvestia, Atmospheric and Oceanic Physics, Vol. 43, No. 6, 2007, pp. 783-791. doi:10.1134/S0001433807060060

[12] G. I. Marchuk, "The Numerical Solution of the Problems of Dynamics of the Atmosphere and Ocean,” Hydrometeoizdat, Leningrad, 1974 (in Russian).

[13] L. N. Gutman, "Introduction to the Nonlinear Theory of Mesometeorological Processes in the Atmosphere," Hydrometeoizdat, Leningrad, 1969 (In Russian).

[14] L. T. Matveev, "The Course of General Meteorology, Physics of Atmosphere and Ocean,” Hydrometeoizdat, Leningrad, 1984 (In Russian).

[15] A. F. Chudnovski, "Heat Physics of Soils,” Nauka, Moscow, 1976 (In Russian).

[16] G. I. Marchuk, V. P. Dimnikov, V. B. Zalesnii, V. N. Likosov and V. Ya. Galin, "The Mathematical Simulation of General Circulation of the Atmosphere and Ocean,” 
Hydrometeoizdat, Leningrad, 1984 (In Russian).

[17] P. N. Belov, E. P. Borisenkov and B. D. Panin, "The Numerical Methods of Weather Forecast,” Hydrometeoizdat, Leningrad, 1989 (In Russian).

[18] F. G. Shuman and L. R. Hovermale, "An Operational Six-Layer Primitive Equation Model,” Journal of Applied Mechanics, Vol. 7, No. 4, 1968, pp. 525-547.

[19] E. Palmen and C. W. Newton, "Atmospheric Circulation Systems”. Academic Press, New York and London, 1969.

[20] S. S. Zilitinkevich and A. S. Monin, "The Turbulence in Dynamical Models of the Atmosphere,” Nauka, Leningrad, 1971.

[21] G. I. Marchuk, V. P. Kochergin, A. S. Sarkisyan et al., "Mathematical Models of Ocean Circulation," Nauka, Novosibirsk, 1980.

[22] A. L. Kazakov and G. L. Lazriev, “About Parameterization of the Surface Layer of the Atmosphere and Active Layer of the Soil," Izvestia AN SSSR, Phizika Atmosfery $i$ Okeana, Vol. 14, No. 3, 1978, pp. 257-265.

[23] K. I. Papinashvili, "Atmospheric Processes in Transcaucasia and Their Relation with Macrocirculation Processes Above the Territory of Eurasia,” Hydrometeoizdat, Leningrad, 1963, p. 184.

[24] T. W. Troutman, D. B. Elson and M. A. Rose, "A Severe Weather Threads Checklist to Determine Pre-Storm Environment," www.srh.noaa.gov/ohx/?n=checklist

[25] "Bulk Richardson Number," Wikipedia, the free ency- clopedia.

http://www.mythical-buddies.com/index.php?q=Bulk_Ri chardson_number

[26] M. Kordzakhia, “The Climate of Georgia,” Publishing House of the Georgian Academy of Sciences, Tbilisi, 1961.

[27] "The Atlas of Excitement and a Wind of the Black Sea," Gidrometeoizdat, Leningrad, 1969.

[28] "The Climate and Climatic Resources of Georgia," Gidrometeoizdat, Leningrad, 1971.

[29] Yu. D. Chashechkin, "Stochasticity of Convective Flows in a Stratified Liquid. Non-Linear Waves: Stochasticity and Turbulence," Gorky: AN SSSR, IPF, 1980, pp. 131139.

[30] E. V. Thrane et al., "Neutral Air Turbulence in the Upper Atmosphere Observed during the Energy Budget Campaign,” Journal of Atmosphere and Terrestrial Physics, Vol. 47, No. 1, 1985, pp. 243-264. doi:10.1016/0021-9169(85)90136-9

[31] F. J. Schmidlin, M. Carlson, D. Rees, D. Offermann, Philbrick and H. U. Widdel, "Wind Structure and Variability in the Middle Atmosphere during the November 1980 Energy Budget Campaign,” Journal of Atmosphere and Terrestrial Physics, Vol. 47, 1985, pp. 183-193. doi:10.1016/0021-9169(85)90133-3

[32] A. I. Gvelesiani and A. T. Eliava, "On the Subrange of Buoyancy of the Turbulent Mesosphere,” Izv. RAN, FAO, Vol. 34, No. 2, 1998, pp. 241-244. 